ISSN electrónico: 2172-9077

DOI: https://doi.org/10.14201/fjc-v22-26275

\title{
INNOVACIÓN EN LA COMUNICACIÓN ESTRATÉGICA
}

\section{Innovation in strategic communication}

\author{
Ph. D. Verónica ALTAMIRANO
}

Docente. Universidad Técnica Particular de Loja, Ecuador

E-mail: vpaltamirano@utpl.edu.ec

(iD) https://orcid.org/0000-0003-1238-1886

Mgt. Rosario PUERTAS HIDALGO

Docente. Universidad Técnica Particular de Loja, Ecuador

E-mail: rjpuertas@utpl.edu.ec

(iD) https://orcid.org/0000-0002-9545-2223

\author{
Ph.D. Jenny YAGUACHE QUICHIMBO \\ Docente. Universidad Técnica Particular de Loja, Ecuador \\ E-mail: jjyaguache@utpl.edu.ec \\ (iD) https://orcid.org/0000-0002-5489-4228
}

Fecha de recepción del artículo: 20/03/2021

Fecha de aceptación definitiva: 30/03/2021

La comunicación estratégica evoluciona, permanentemente, para adaptarse a los cambios de la sociedad. Pasamos de una comunicación de masas controlada, basada en la publicidad y la promoción asimétrica y unidireccional, a una nueva era de la información multimediática en la Red, que busca fortalecer las relaciones entre cliente y empresa. Esta evolución se caracteriza por la participación de los clientes y por el surgimiento de un sin número de pequeñas y medianas empresas on line que han conseguido posicionarse en el mercado gracias a las Tecnologías de la Información y la Comunicación y que son creadas para satisfacer las necesidades del nuevo cliente, que exige, cada vez más, un producto personalizado.

Desde siempre, en el proceso de decisión y compra de un producto o servicio el cliente busca información y referencias de la oferta (Altamirano, Ochoa y Túñez, 2018, pero, este proceso de comunicación, en la actualidad, está influenciado por una actitud colaborativa de los usuarios, que comparten voluntariamente sus opiniones y experiencias con las marcas en las redes sociales, lo que implica comunicación bidireccional e interactiva en planos de igualdad por ambas partes.

En este escenario, los sitios web se consolidan como una plataforma indispensable para la promoción y comunicación organizacional, pero los cambios en el comportamiento del usuario 2.0, exigen que estas plataformas se adapten a sus necesidades y 
ofrezcan recursos y herramientas que dinamicen la oferta junto a espacios de interactividad que permitan su participación y el establecimiento de una asesoría directa en la red. Estos sitios se complementan con la presencia de los social media, que contribuyen a generar espacios para la co creación de contenidos y consecuentemente para la generación de una comunicación transmedia, ha que tener presente que "habrá fidelizacion de clientes a partir de la buena experiencia” (Pérez, Hoyos, Alzate, y Arroyave, 2019).

Es decir, la forma como se comunica la sociedad ha evolucionado a un modelo bidireccional, participativo, voluntario y comprometido, en el que el usuario es quien controla los procesos de comunicación.

Estos procesos continúan innovando, y surge la comunicación 3.0, que se basa en la Web Semántica, que consiste en analizar los datos que aportan, voluntariamente, los usuarios para conocer su comportamiento, preferencias, gustos, necesidades, esta información permite conocer mejor el mercado y al cliente para personalizar la comunicación y ofrecer productos y servicios a la medida, lo que constituye un avance significativo en la comunicación estratégica. La influencia del big data permite evolucionar las predicciones modeladas, esto es, la capacidad, gracias al estudio avanzado de los datos, de predecir y modelar los productos y comunicaciones que serán eficaces en el futuro (Navío-Navarro, 2020).

Y finalmente, avanzamos hacia la automatización de los procesos la inclusión de la inteligencia artificial para mejorar la atención al cliente lo que permite fortalecer las relaciones cliente - empresa, que es el objetivo de la comunicación estratégica,

El monográfico Innovación en la Comunicación Estratégica presenta estudios e investigaciones referentes a los avances en el ámbito de la comunicación organizacional, por esta razón abrimos el monográfico con la descripción y el análisis del contexto actual en el que se desarrolla la comunicación estratégica, en el que explica los procesos de innovación y automatización de la comunicación, este capítulo introductorio es presentado por el Miguel Túñez López.

Se continúa analizando una de las estrategias con mayor impacto en los últimos tiempos como es la incorporación de los influencers en la estrategia de comunicación. En el artículo "Impacto de los influencers en las estrategias promocionales del sector gastronómico de la ciudad de Medellín" escrito por Sandra Milena Amaya Henao, Germán Augusto Silva Cortés y Yanyn Aurora Rincón Quintero, se concluye que el impacto del influencer en las estrategias promocionales para el sector gastronómico de la ciudad de Medellín es altamente favorable porque los influencers son la opción predilecta cuando los establecimientos del sector desean promocionar sus productos, ya que logran un mayor alcance en menos tiempo y a menor costo que con otros medios más tradicionales,

Hablando de los resultados en la implementación de estrategias digitales en la comunicación, David Parra Valcarce y Charo Onieva Mallero, evalúan cómo aportan las redes sociales al incremento del volumen de tráfico web hacia los portales de los cibermedios nativos digitales, la investigación "Análisis del impacto de las redes sociales sobre el tráfico web de los cibermedios nativos digitales españoles" se sustenta en aspectos como la consolidación de la web semántica manual o la eclosión de los prosumidores.

Uno de los cambios más significativos en el campo de la comunicación, es la forma de consumir contenido audiovisual en todo el mundo, esto se demuestra en el artículo "Posicionamiento en entornos digitales: el caso de Netflix y su interacción 
con los públicos", escrito por María Jesús Fernández Torres y Eduardo Villena Alarcón, que concluyen que Netflix no solo ha revolucionado la forma en la que se accede a los contenidos audiovisuales, sino que también ha innovado en la forma de compartir contenidos a través de redes sociales para posicionar sus productos en la mente de sus suscriptores.

La migración a las plataformas digitales requiere adaptarse a las nuevas narrativas digitales, por esta razón, El estudio "Narrativas transmedia y proximidad política en la campaña electoral de Jorge Yunda Machado, alcaldía de quito 2019" explora cómo se utilizó la narrativa transmedia para generar estrategias de proximidad en la campaña municipal de Jorge Yunda Machado, y cómo éstas condicionaron la imagen del candidato, como un político "cercano y humano» permitiendo así una identificación "anti carismática" hacia la comunidad de electores, esta investigación la realizaron María José Enríquez Cruz y Juan Sebastián Gómez Navas.

En este sentido, también es importante el mensaje que se transmite a las diferentes audiencias, teniendo mayor relevancia cuando se trabaja en el ámbito de la salud. Los resultados de la investigación "Ciencia para todos: guías para superar los desafíos de la comunicación científica en casos de comunicación de la salud" se evidencian la importancia del involucramiento de los investigadores como voceros; criterios de diseño de mensajes para no-expertos; y criterios para seleccionar canales comunicacionales. Denisse Vásquez-Guevara presenta una guía práctica para comunicar de manera idónea los mensajes de salud.

Laura García-Huguet en el artículo, "La comunicación online de la RSC en materia medioambiental. el caso de empresas de moda rápida y moda lenta", afirma que la preocupación de la ciudadanía por el impacto medioambiental de sus acciones los ha llevado a replantear sus hábitos de consumo hacia unos más sostenibles con su entorno.

Finalmente, los invitamos a leer estas interesantes investigaciones que analizan la comunicación estratégica desde una perspectiva innovadora.

\section{Bibliografía}

Altamirano, V., Ochoa, S. y Túñez, Mi. (2018) "Modelos para evaluar la comunicación 2.0 en sitios web y redes sociales organizacionales", 2018 XIII Congreso Ibérico de Sistemas y Tecnologías de la Información (CISTI), Cáceres, 2018, pp. 1-6, doi: 10.23919/CISTI.2018.8399432.

Navío-Navarro, M. (2020) Las redes sociales como elemento dinamizador de la evolución de la comunicación publicitaria: del marketing 1.0 al marketing 3.0. Las redes sociales como herramienta de comunicación persuasiva, en Liberal Ormaechea y Mañas Viniegra (coord..), págs. 795-831

Pérez, F. Hoyos, D. Alzate, M. y Arroyave, L. M. A. (2019). Impacto del comercio electrónico en las redes sociales sobre la decisión de compra en ciudadanos asentados en el Valle de Aburrá. RHS: Revista Humanismo y Sociedad, 7(2), 26-40. 